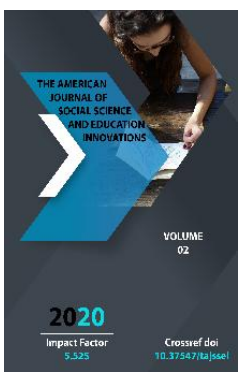

\section{The Effect Of The Introduction Of Foreign Languages Into The Specialty}

\author{
Nafisakhon Minovarovna Kazimova \\ Teacher Of Chemistry, Associate Professor, Kokand State Pedagogical Institute Named After \\ Mukimi, Uzbekistan
}

Journal Website: http://usajournalshub.c Shakhzodakhon Madaminjon Qizi Kodirova (Mukhtorova) om/index,php/tajssei Master Of Chemistry Teaching Methods Kokand State Pedagogical Institute Named After

Copyright: Original content from this work may be used under the terms of the creative commons attributes 4.0 licence.

\title{
ABSTRACT
}

This article is devoted to the effect of the introduction of foreign languages in the specialty, the study of namingsome terms in English in the process of studying the specialty sciences.The article provides scientific information on chemistry, analytical chemistry in English. The conclusion is given at the end of the article.

\section{KEYWORDS}

Chemistry, branches of chemistry,analytical chemistry,qualitative analysis,quantitative analysis, separate, wet chemical methods, instrumental methods, Gravimetric methods, Volumetric methods, electroanalytical methods,spectroscopic methods

\section{INTRODUCTION}

All the students welcomed the 5 initiatives put forward by the President of Uzbekistan Sh.M.Mirziyoev and are trying to contribute to each of them.In particular, there are some positive changes in students in computer literacy and language learning.

Nowadays, in the field of education,especially in higher education,there is a strong demand 
for the study of foreign languages,along with the study of the subjects in which he is interested.There is a lot of work being done among students, especially gifted students, to learn the language and apply it to their specialization.

There are many benefits to learning some of the terms in English as you study the subject. Even a student who doesn't know English at all can gradually learn these terms through dictionaries, and this is a useful activity. In particular,in the field or analytical chemistry "Quality" and "Quantitative" analysis is studied in two parts.Its practical methods are based on the needs of primary chemical production.

\section{MAIN PART}

Chemistry is the study of matter, including its composition,

structure, physical properties, and reactivity.

Chemistry is the study of matter and energy and the interactions between them. Chemistry and physics are specializations of physical science. Chemistry tends to focus on the properties of substances and the interactions between different types of matter, particularly reactions that involve electrons.

Chemistry is the science that systematically studies the composition, properties, and activity of organic and inorganic substances and various elementary forms of matter.

\section{Why Study Chemistry?}

Because understanding chemistry helps you to understand the world around you. Cooking is chemistry. Everything you can touch or taste or smell is a chemical. When you study chemistry, you come to understand a bit about how things work. Chemistry isn't secret knowledge, useless to anyone but a scientist.
It's the explanation for everyday things, like why laundry detergent works better in hot water or how baking soda works or why not all pain relievers work equally well on a headache. If you know some chemistry, you can make educated choices about everyday products that you use.

\section{What Fields of Study Use Chemistry?}

You could use chemistry in most fields, but it's commonly seen in the sciences and in medicine. Chemists, physicists, biologists, and engineers study chemistry. Doctors, nurses, dentists, pharmacists, physical therapists, and veterinarians all take chemistry courses. Science teachers study chemistry. Fire fighters and people who make fireworks learn about chemistry. So do truck drivers, plumbers, artists, hairdressers, chefs... the list is extensive.

\section{What Do Chemists Do?}

Whatever they want. Some chemists work in a lab, in a research environment, asking questions and testing hypotheses with experiments. Other chemists may work on a computer developing theories or models or predicting reactions. Some chemists do field work. Others contribute advice on chemistry for projects. Some chemists write. Some chemists teach.

\section{Branches Of Chemistry}

Physical Chemistry - study of the physical and fundamental basis of chemical systems and processes. In particular, the energetics and dynamics of such systems and processes are of interest to physical chemists. Important areas of study include chemical thermodynamics, chemical kinetics, electrochemistry, statistical mechanics, spectroscopy, and more recently, astrochemistry. 
Organic Chemistry - study of the structure, properties, composition, mechanisms, and reactions of organic compounds.

(Biochemistry, Medicinal chemistry, Pharmacy, ...)

Inorganic Chemistry - study of the properties and reactions of inorganic compounds.

Nuclear Chemistry - study of how subatomic particles come together and make nuclei.

Analytical Chemistry - analysis of material samples to gain an understanding of their chemical composition and structure. Analytical chemistry incorporates standardized experimental methods in chemistry.

Biochemistry - The study of the chemistry inside living organisms. Biochemistry includes the study of Immunology, Molecular Biology, and Neurochemistry.

Other: agrochemistry, molecular biology, nanotechnology, petrochemistry, pharmacology, ...

Analytical chemistry is often described as the area of chemistry responsible for

Characterizingthe composition of matter, both qualitatively (what is present) andquantitatively (how much is present).

Analytical chemistry studies and uses instruments and methods used to separate, identify, and quantify matter.In practice separation, identification or quantification may constitute the entire analysis or be combined with another method. Separation isolates analytes. Qualitative analysis identifies analytes, while quantitative analysis determines the numerical amount or concentration.

Analytical chemistry consists of classical, wet chemical methods and modern, instrumental methods.Classical qualitative methods use separations such as precipitation, extraction, and distillation.

Analytical chemistry has been important since the early days of chemistry, providing methods for determining which elements and chemicals are present in the object in question. During this period significant contributions to analytical chemistry include the development of systematic elemental analysis by Justus von Liebig and systematized organic analysis based on the specific reactions of functional groups.

The first instrumental analysis was flame emissive spectrometry developed by Robert Bunsen and Gustav Kirchhoff who discovered rubidium (Rb) and caesium(Cs) in 1860.

\section{Qualitative Analysis}

A qualitative analysis determines the presence or absence of a particular compound, but not the mass or concentration. By definition, qualitative analyses do not measure quantity.

\section{Quantitative Analysis}

- Gravimetric methods determine the mass of the analyte or some compound chemically related to it.

- Volumetric method, the volume of a solution containing sufficient reagent to renact completely with the analyte is measured.

- Electroanalytical methods involve the measurement of such electrical properties as voltage, current, resistance, and quantity of electrical charge. 
- Spectroscopic method sare based on measurement of the interaction between electromagnetic radiation and analyte atoms or molencules or on the production of such radiation by analytes. Finally, there is a group of miscellaneous methods that includes the measurement of such quantities as mass-to-charge ratio of molecules by mass spectrometry, rate of radioactive de $n$ cay, heat of reaction, rate of reaction, sample thermal conductivity, optical activity, and refractive index.

- Qualitative analysis is what.

- Quantitative analysis is how much.

Chemical Analysis Affects Many Fields

- Manufacturing:

- Quality control of packaged foods specifications

- Forensics:

- Chemical features for criminal evidence

Role of Analytical Chemistry in Modern Science

- Case Study 1.

- Nuclear Waste Disposal

- Nuclear Power Plants

- Nuclear Reactors

- Weapons Processing

- Weapons Disposal

\section{CONCLUSION}

The article was reviewed by students and teachers, opinions and comments were expressed.It was mentioned that through this method, student's interest in science and a foreign language can be further increased.
This means that student can learn language in an integral way with science.This gives a useful and effective result at the same time.

\section{REFERENCES}

1. Rasulov K.R. Analitik kimyo.Toshkent, 2004.

2. Gulamova M.T., Norov Sh.Q. Analitik kimyo. Toshkent, 2009.

3. David Harvey. Modern Analytical Chemistry. DePauw University. 